\title{
Consumo de energía y emisiones de bióxido de carbono del sector refinación de petróleo en México de 2015 a 2030
}

\section{Energy Consumption and Carbon Dioxide Emissions from Petroleum Refining Sector in Mexico from 2015 to 2030}

\author{
Granados-Hernández Elías \\ Universidad Nacional Autónoma de México \\ Laboratorio de Ingeniería Ambiental \\ Centro Tecnológico Aragón \\ Correo:elias78@unam.mx \\ Bravo-Álvarez Humberto \\ Universidad Nacional Autónoma de México \\ Sección de Contaminación Ambiental \\ Centro de Ciencias de la Atmósfera \\ Correo:hbravo@unam.mx \\ Sosa-Echeverría Rodolfo \\ Universidad Nacional Autónoma de México \\ Sección de Contaminación Ambiental \\ Centro de Ciencias de la Atmósfera \\ Correo:rodsosa@unam.mx
}

\author{
López-Andrade Xicoténcatl \\ Universidad Nacional Autónoma de México \\ Laboratorio de Sintesis de Materiales \\ Centro de Física Aplicada y Tecnología Avanzada \\ Correo:xico@fata.unam.mx \\ García-Landa Claudia \\ Universidad Nacional Autónoma de México \\ Correo:dispensario@hotmail.com \\ Sánchez-Álvarez Pablo \\ Universidad Nacional Autónoma de México \\ Sección de Contaminación Ambiental \\ Centro de Ciencias de la Atmósfera \\ Correo:pasa@unam.mx
}

Información del artículo: recibido: abril de 2014, reevaluado: agosto de 2014, aceptado: abril de 2015

\section{Resumen}

El incremento en el volumen de producción de petrolíferos de alta demanda, como la gasolina, eleva el uso de energía y, en consecuencia, las emisiones de $\mathrm{CO}_{2}$ en las refinerías de petróleo. En México, por ejemplo, el escenario de demanda de gasolinas para los próximos 20 años considerando una tendencia histórica en el crecimiento vehicular aumentará el consumo de este combustible en casi 55\%. El propósito de este trabajo es conocer el impacto de consumo de energía por unidad de crudo procesado y las emisiones de $\mathrm{CO}_{2}$ utilizando la metodología del Panel Intergubernamental para el Cambio Climático (PICC), así como el empleo del cálculo estequiométrico con base en el contenido de carbono. Se realizaron diferentes proyecciones empleando cuatro opciones tecnológicas de refinación de petróleo procesando tres tipos de crudos puros (Olmeca, Istmo y Maya) y cuatro mezclas (M1, M2, M3, M4). Al realizar un balance energético los resultados muestran que proyectar refinerías muy complejas para cubrir una demanda específica de gasolinas consumirá menos energía y por consiguiente las emisiones de $\mathrm{CO}_{2}$ serán menores. Este estudio es relevante como herramienta de planeación energética y de contaminación ambiental en México, así también, como objeto de análisis del sector energético a nivel mundial.

\section{Descriptores:}

- proyecciones de refinación

- consumo de energía

- emisiones de bióxido de carbono

- tipos de refinería

- unidades de proceso

- tipos de petróleo crudo 


\begin{abstract}
The increase in the volume of production of petroleum products of high demand, such as gasoline, rises the use of energy and therefore emissions of $\mathrm{CO}_{2}$ in oil refineries. In Mexico, for example, gasoline demand scenario for the next 20 years growth will increase the fuel consumption by almost 55\%, considering a historical trend in traffic. The purpose of this study as to determine the impact of energy consumption per unit of processing oil and $\mathrm{CO}_{2}$ emissions using the methodology of the Intergovernmental Panel on Climate Change (IPCC) as well as employing the stoichiometric calculation based on the carbon content. Different projections were made using four technological options of oil refining, processing three types of pure raw (Olmeca, Istmo, Maya) and four blends (M1, M2, M3, M4). When performing an energy balance results show that projecting very complex refineries to meet a specific demand for gasoline will consume less energy and therefore $\mathrm{CO}_{2}$ emissions will be lower. This study is important as a tool for energy planning and environmental pollution in Mexico, as well as an object of analysis of the energy sector worldwide.
\end{abstract}

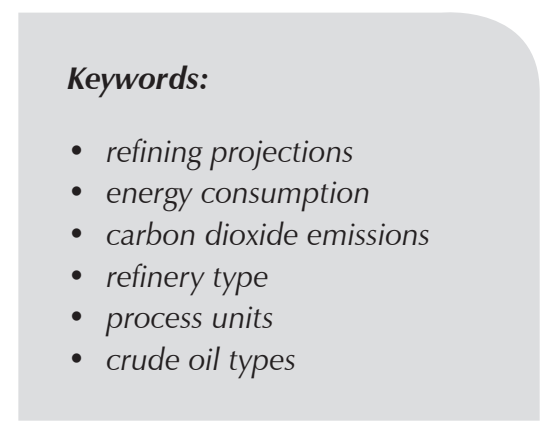

\section{Introducción}

El crecimiento económico de un país se relaciona estrechamente con el consumo de energía y emisiones de bióxido de carbono $\left(\mathrm{CO}_{2}\right)$, pues, a mayor energía utilizada, mayor crecimiento económico. Así también, el uso más eficiente de la energía podría entrañar crecimiento económico y una reducción en el uso de energía y de las emisiones de $\mathrm{CO}_{2}$. La eficiencia energética puede constituir la fuerza motriz económica de un país (Grid, 2014).

A nivel mundial el consumo total de energía para el año 2012 fue de 13157 millones de toneladas equivalentes de petróleo (MMtep), 1.4\% más con respecto al año 2011 que fue de 12981 MMtep y 2.2\% más con relación al año 2000 que fue de 10101 MMtep (Enerdata, 2013). Con proporción solamente al consumo total de energía primaria, el incremento fue de $2.1 \%$ y $2.4 \%$, respectivamente, es decir, se consumieron 252 MMtep más que en el año 2011 y 3137 MMtep más que en el 2000 (BP Statistical Review of World Energy June, 2013). El porcentaje promedio de consumo de energía primaria de acuerdo con el total consumido en el periodo del año 2000 al 2012 fue de 93\%. Es decir, la energía primaria es la fuente más importante de uso.

En el Sector Refinación de Petróleo (SRP) una gran cantidad de esta energía se utiliza para el procesamiento de crudo, garantizando todos los servicios requeridos para sus operaciones por medio de las diferentes transformaciones simples o complejas, utilizando parte de los productos obtenidos. Así, un indicador de eficiencia energética en las refinerías de petróleo, es una parte proporcional del mismo crudo procesado que se utiliza para satisfacer la demanda de energía que necesita dicha refinería para su funcionamiento.
Actualmente en las refinerías de petróleo, la proporción de crudo utilizado para la generación de energía está entre $4 \%$ y $8 \%$, y esta puede ser menor o mayor, dependiendo del nivel de complejidad de la refinería. Este incremento por el nivel de complejidad de la refinería de petróleo se puede expresar como:

a) Parte del consumo de energía de la cantidad total de petróleo crudo procesado,

b) consumo de energía específica por tonelada de petróleo crudo procesado y

c) por tonelada de productos refinados (Ozren, 2005).

De acuerdo con la Agencia Internacional de Energía (IEA), el consumo mundial de energía en las refinerías de petróleo para el año 2011 fue de 273 MMtep, cuatro MMtep más que en 2010 (IEA, 2013a) y siete MMtep más que en 2009 (IEA, 2012a), pero cuatro MMtep menos que en 2008 (IEA, 2011a). Del total consumido, el promedio de utilización en los años 2008, 2009, 2010 y 2011 para los países no integrantes de la Organización para la Cooperación y el Desarrollo Económico (OECD), fue de 43\% (IEA, 2011a, 2012a y 2013a) y 55\% para los que integran este organismo (IEA, 2011b, 2012b y 2013b). En América del Norte, México como país miembro de la OECD participó con 3\% del promedio usado en los años que se mencionaron anteriormente, al igual que Canadá, pero Estados Unidos (EU) es el mayor consumidor con $25 \%$.

Por otra parte, las emisiones mundiales de $\mathrm{CO}_{2}$ por la combustión de combustibles fósiles alcanzaron un nivel récord de 31.6 giga toneladas (Gt) en 2011, según las primeras estimaciones de la IEA (2013c). Esto representa un aumento de $1.0 \mathrm{Gt}$ con respecto al año 2010, es decir, un incremento de $3.2 \%$. El carbón contribuyó con 
$45 \%$ de las emisiones totales de $\mathrm{CO}_{2}$ relacionadas con la energía en 2011, seguido por el petróleo (35\%) y gas natural (20\%). En 2012, un incremento de 2.1\% en las emisiones de $\mathrm{CO}_{2}$ de acuerdo con el año 2011 en países no integrantes de la OECD, fue solo parcialmente compensado por una reducción de $1 \%$ en las emisiones de países integrantes de la OECD. China realizó la mayor contribución al incremento global aumentando en 548.4 millones de toneladas (MMt), es decir, registró un incremento de $6.3 \%$. América del Norte, presentó una reducción de $2.8 \%$ debido principalmente a que EU mostró una disminución de $3.6 \%$ y Canadá $0.7 \%$ con 216.6 y $4.1 \mathrm{MMt}$, respectivamente. México por su parte incrementó su emisión 4.6\% con 21.8 MMt (BP, 2013).

De acuerdo con datos de la IEA (IEA 2010a, 2011c, 2012c) las emisiones de $\mathrm{CO}_{2}$ en la industria energética, la cual incluye a la industria de refinación de petróleo, muestran que la mayor emisión de este contaminante se debe al petróleo, que registró un total de 191.5 MMt en 2010, 2.2 MMt menos que en 2009 y 7.3 MMt menos que en 2008. EU participó con un promedio en estos años de $74.7 \%$, Canadá con $15.5 \%$ y México con $9.8 \%$. Considerando lo anterior, es preciso realizar un análisis de diferentes proyecciones del SRP en México, que permitan cumplir con el objetivo principal de este trabajo, que es conocer el impacto del consumo energético por unidad de crudo procesado, así como las emisiones de $\mathrm{CO}_{2}$ utilizando la metodología del PICC, y empleando el cálculo estequiométrico con base en el contenido de carbono por tipo de crudo o mezcla estudiada.

\section{Desarrollo}

Las diferentes proyecciones obtenidas para este análisis se determinaron utilizando opciones tecnológicas de la refinación de petróleo analizadas en Granados et al. (2013), recordando que R1 se refiere a una refinería simple (hydroskimming), R2 a una semi compleja (hydrocracking), R3 a una compleja (catalytic cracking) y R4 a una muy compleja (full conversion), utilizando tres tipos de crudos (Olmeca, Istmo y Maya) y cuatro mezclas de los de mayor reserva Istmo-Maya (\%): M1 (70-30), M2 (6535), M3 (50-50) y M4 (44-56), calculando el volumen de crudo para cada proyección desarrollada en esta investigación. Asimismo, la capacidad de carga de los procesos característicos de las mismas para cada escenario de demanda. En este tenor y debido a la cantidad de datos obtenidos, todo resultado que se muestra en este estudio consideró solamente al crudo Maya. Cualquier cálculo para otro tipo de crudo puro o mezcla se realizará de la misma manera (el mismo algoritmo) que con el crudo Maya. El consumo de energía se presenta en uni-

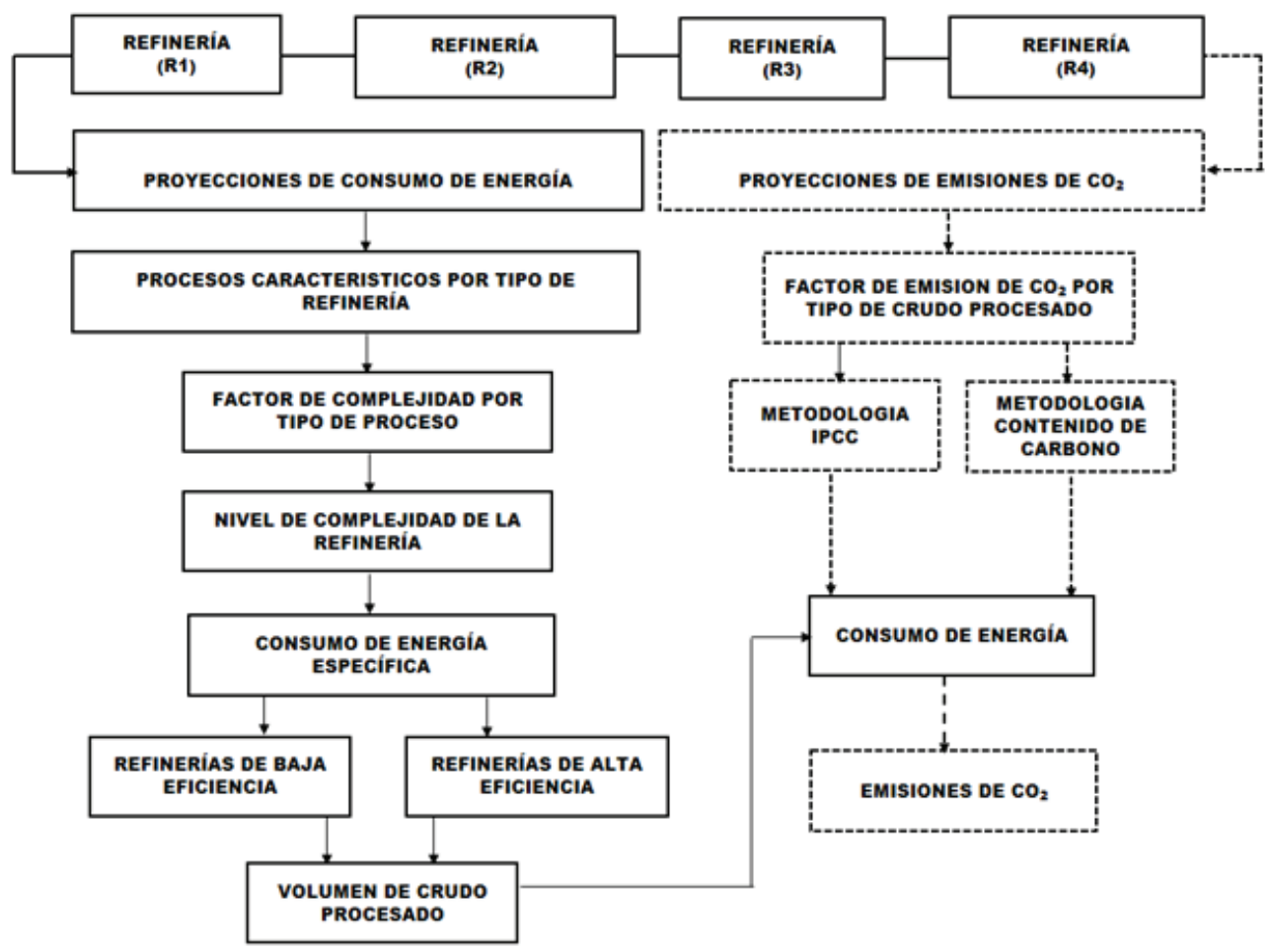

Figura 1. Diagrama de flujo de la metodología para las diferentes proyecciones de consumo de energía y emisiones de $\mathrm{CO}_{2}$ 
dades de miles de toneladas equivalentes de petróleo diario (Mtepd) y las emisiones de $\mathrm{CO}_{2}$ en miles de toneladas diarias (Mtd). Por otro lado, los datos requeridos de capacidad de carga y volumen de crudo se presentan en Mtd. Para este estudio se utilizaron hojas de cálculo Excel siguiendo el algoritmo de cómputo utilizado en la publicación previa (Granados et al., 2013), adicionando las variables y realizando los cálculos de interés para esta investigación. La figura 1 muestra el procedimiento metodológico para este trabajo, las líneas continuas en los bloques, después de indicar las refinerías, muestran el procedimiento para determinar el consumo de energía, y los bloques punteados el cálculo para obtener las emisiones de bióxido de carbono.

\section{Consumo de energía}

Determinar el consumo de energía requirió primero de calcular el nivel de complejidad de cada refinería con base en los procesos que las caracterizan y utilizando su capacidad de carga. De esta manera, como datos requeridos para este cálculo, la tabla 1 presenta el factor de complejidad por tipo de proceso para cada opción tecnológica y la tabla 2 muestra la capacidad de carga para un escenario tendencial (BAU) para el año 2015 (como ejemplo), con base en la tendencia histórica anual del crecimiento vehicular. Así también, se muestran tres escenarios inflacionarios basados en el crecimiento poblacional y en el Producto Interno Bruto (PIB) denominados alto, bajo y de referencia (RAG-A, RAG-B, RAG-C) (Granados et al., 2013).

Tabla 1. Procesos característicos de diferentes tipos de refinerías y factor de complejidad para cada proceso

\begin{tabular}{|c|c|c|c|c|c|}
\hline Procesos & $\mathrm{R} 1$ & $\mathrm{R} 2$ & $\mathrm{R} 3$ & $\mathrm{R} 4$ & $\begin{array}{c}\text { Factor de } \\
\text { Complejidad }\end{array}$ \\
\hline $\begin{array}{l}\text { Destilación Atmosférica } \\
\text { (DA) }\end{array}$ & ---- & ---- & ---- & ---- & 1 \\
\hline $\begin{array}{l}\text { Hidrotratador de Naftas } \\
(\mathrm{HN})\end{array}$ & ---- & ---- & ---- & ---- & 2 \\
\hline $\begin{array}{l}\text { Reformador Catalítico } \\
\text { (RC) }\end{array}$ & ---- & ---- & ---- & ---- & 5 \\
\hline $\begin{array}{l}\text { Hidrotratador de } \\
\text { Destilados (HD) }\end{array}$ & ---- & ---- & ---- & ---- & 7 \\
\hline $\begin{array}{l}\text { Destilación al Vacío } \\
\text { (DV) }\end{array}$ & & --- & --- & ---- & 2 \\
\hline $\begin{array}{l}\text { Craqueo Catalítico Fluido } \\
(\mathrm{CCF})\end{array}$ & & & ---- & ---- & 6 \\
\hline $\begin{array}{l}\text { Alquilación } \\
\text { (AQ) }\end{array}$ & & & ---- & ---- & 11 \\
\hline $\begin{array}{l}\text { Coquización } \\
\text { (CQ) }\end{array}$ & & & & ---- & 5.5 \\
\hline Fuente: (Baird, 1996) & & & & & $\begin{array}{c}\text { Fuente: } \\
\text { (EPA, 1997) }\end{array}$ \\
\hline
\end{tabular}

Nota: $\mathrm{R} 1=$ refinería simple, $\mathrm{R} 2=$ refinería semi compleja, $\mathrm{R} 3=$ refinería compleja y $\mathrm{R} 4=$ refinería muy compleja
Tabla 2. Capacidad de carga por tipo de proceso, tipo de refinería, procesando crudo Maya y considerando un déficit de gasolinas en el año 2015

\begin{tabular}{cccccccccc}
\hline & \multicolumn{8}{c}{ Miles de toneladas diarias (Mtd) } \\
\cline { 2 - 9 } Refinería Escenario & DA & HN & RC & HD & DV & CCF & AQ & CQ \\
\hline R1 & 367 & 77 & 59 & 66 & -- & --- & --- & -- \\
R2 & BAU & 244 & 71 & 66 & 32 & 105 & --- & --- & --- \\
R3 & 168 & 32 & 25 & 20 & 72 & 47 & 7 & --- \\
R4 & 103 & 34 & 29 & 12 & 44 & 29 & 7 & 15 \\
\hline
\end{tabular}

Fuente: (Granados, 2013)

Nota: $\mathrm{R} 1=$ refinería simple, $\mathrm{R} 2=$ refinería semi compleja, $\mathrm{R} 3=$ refinería compleja y $\mathrm{R} 4=$ refinería muy compleja, $\mathrm{BAU}=$ Escenario Tendencial, DA=Destilación Atmosférica, $\mathrm{HN}=$ Hidrotratador de Naftas, $\mathrm{RC}=$ Reformador Catalítico, $\mathrm{HD}=$ Hidrotratador de Destilados DV=Destilación al Vacío, $\mathrm{CCF}=$ Craqueo Catalítico Fluido, $\mathrm{AQ}=$ Alquilación, $\mathrm{CQ}=$ Coquización

El nivel de complejidad para cada opción tecnológica se obtuvo utilizando la siguiente relación matemática

$N C r=\sum_{i=1}^{n}\left(f_{\mathcal{C}_{1}} *\left[\frac{C c i}{C d a}\right]\right)$

Donde:

$N c r=$ Nivel de complejidad de la refinería

$i=$ Tipo de proceso

$f c i=$ Factor de complejidad

$C c i=$ Capacidad de carga del proceso $(\mathrm{Mtd})$

$\mathrm{Cd} a=$ Capacidad destilación atmosférica (Mtd)

Como ejemplo de cálculo se muestra el procedimiento obtenido para la refinería R4 utilizando los datos de las tablas 1 y 2, aplicando la ecuación 1 . NCr $=[1 \times(103 / 103)]$ $+[2 \times(34 / 103)]+[5 \times(29 / 103)]+[7 \times(12 / 103)]+$ $[2 \times(44 / 103)]+[6 \times(29 / 103)]+[11 \times(7 / 103)]+$ $[5.5 \times(15 / 103)]=8$. De esta manera, los resultados del nivel de complejidad para las siguientes opciones tecnológicas de refinación quedarían de la siguiente forma: $\mathrm{R} 1=3.5, \mathrm{R} 2=4.7$ y R3 = 6.0.

El segundo cálculo fue determinar el consumo de energía específica de cada opción tecnológica empleando el nivel de complejidad y datos de la figura 2. De esta manera, se localizó el nivel de complejidad obtenido y se relacionó con un consumo de energía específica. Para este procedimiento se consideraron como variables las refinerías de baja y alta eficiencia (RBE, RAE) como lo muestra la figura 2. Por lo tanto, para este cálculo se emplearon las ecuaciones de tendencia expuestas en dicha figura. Igualmente, como ejemplo del tratamiento de la información se muestra lo realizado 
para la tecnología $\mathrm{R} 4$ : refinería de baja eficiencia $(\mathrm{RBE})=0.6178 \times(8.0)+0.6683=5.6 \mathrm{GJ} / \mathrm{t}$ y refinería de alta eficiencia $(\mathrm{RAE})=0.4425 \times(8.0)+0.0075=3.5 \mathrm{GJ} / \mathrm{t}$. De esta manera, la tabla 3 muestra el consumo de energía específica para cada una de las opciones tecnológicas de refinación de petróleo.

Finalmente, el último cálculo fue determinar el consumo de energía total derivado de multiplicar los resultados de la tabla 3 por el volumen de crudo procesado analizado en cada proyección (Granados et al., 2013). Como ejemplo para este trabajo se presentan los datos para un escenario BAU (R1= 367, R2=244, R3=168, R4=103 en Mtd) procesando crudo Maya y considerando déficit de gasolinas para el año 2015.

En conclusión y como ejemplo, en la figura 3 se muestran el consumo de energía para cubrir una demanda de gasolinas y el porcentaje de consumo con respecto al total de crudo procesado en las tecnologías analizadas en este trabajo para los diferentes escenarios, independientemente del escenario elegido, las tendencias que se muestran son las mismas.
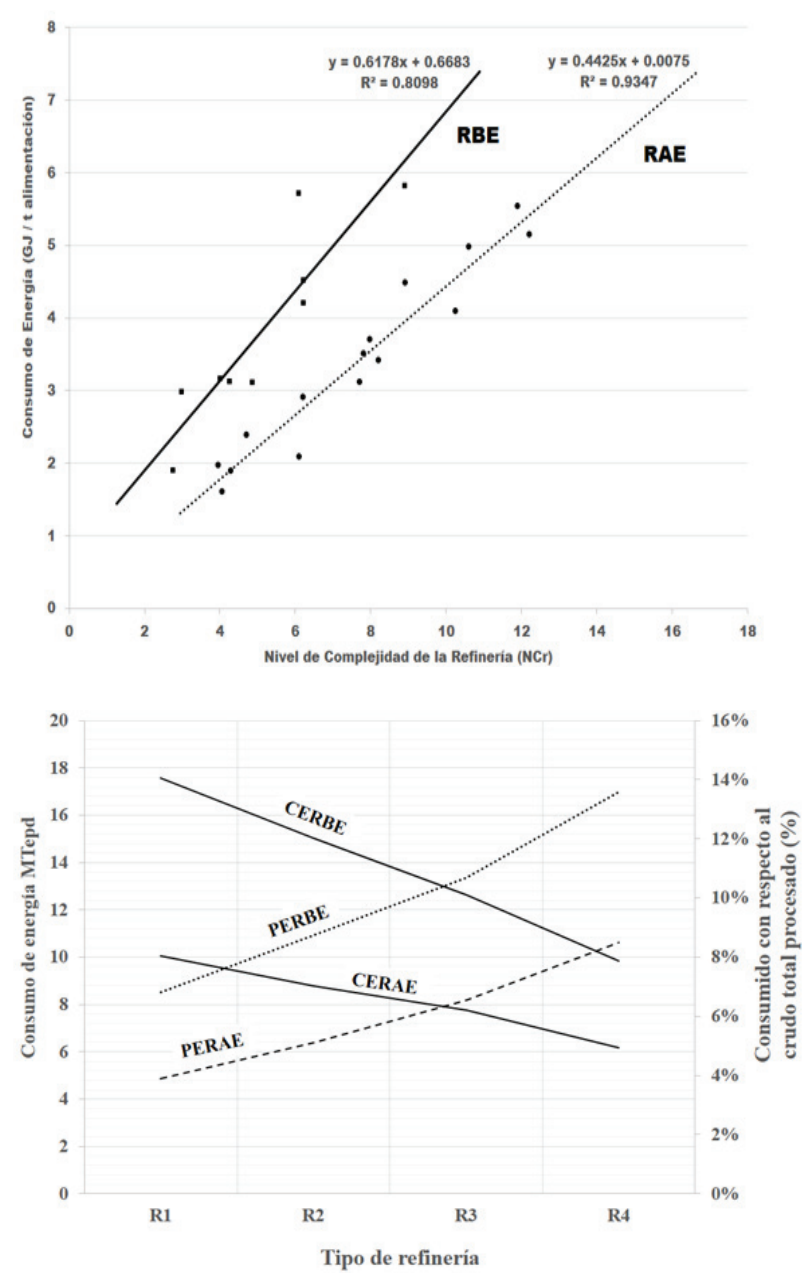

De esta manera y para fines prácticos, en la tabla 4 se muestra el consumo de energía procesando crudo Maya con déficit de gasolinas en RBA y RAE analizados para los diferentes escenarios, y empleando los factores de conversión convenientes, de esta forma se obtuvo el consumo de energía en Mtepd.

\section{Emisiones de bióxido de carbono}

Las emisiones de $\mathrm{CO}_{2}$ se obtuvieron al calcular un factor de emisión por tipo de crudo utilizado, empleando dos opciones de cálculo: a) la metodología del PICC y b) por cálculo estequiométrico por contenido de carbono.

La metodología PICC utiliza las directrices de 2006 para los inventarios nacionales de gases de efecto invernadero, módulo energía (PICC, 2014). Por contenido de carbono el paso determinante para la estimación de $\mathrm{CO}_{2}$ derivado del consumo energía, y como porcentaje del total de crudo analizado en cada proyección, es la conversión de las unidades de carbono a unidades de $\mathrm{CO}_{2}$. El primer paso es encontrar el contenido de carbo-

Figura 2. Dependencia del consumo de energía específica y nivel de complejidad tomando como ejemplo 28 refinerías de EEUU (Ozren, 2005).

Nota: $\mathrm{RAE}=$ Refinería de Alta Eficiencia, $\mathrm{RBE}=$ Refinería de Baja Eficiencia

Figura 3. Balance del consumo de energía específica para cubrir una demanda de gasolinas y para el total de crudo procesado.

CERBE: Consumo de Energía-Refinerías de Baja Eficiencia, CERAE: Consumo de Energía-Refinerías de Alta Eficiencia, PERBE: Porcentaje de Consumo-Refinerías de Baja Eficiencia, PERAE: Porcentaje de Consumo-Refinerías de Alta Eficiencia, $\mathrm{R} 1=$ refinería simple, $\mathrm{R} 2=$ refinería semi compleja, $\mathrm{R} 3=$ refinería compleja y $\mathrm{R} 4=$ refinería muy compleja 
Tabla 3. Consumo de energía específica

Consumo de Energía Específica (GJ /t alimentación)

\begin{tabular}{ccc}
\cline { 2 - 3 } Tipo de Refinería & RBE & RAE \\
\hline R1 & 2.8 & 1.6 \\
R2 & 3.6 & 2.1 \\
R3 & 4.4 & 2.7 \\
R4 & 5.6 & 3.5 \\
\hline
\end{tabular}

Nota: GJ: Giga Joule, t: Tonelada, R1 = refinería simple, $\mathrm{R} 2=$ refinería semi compleja, $\mathrm{R} 3=$ refinería compleja $\mathrm{y}$ $\mathrm{R} 4=$ refinería muy compleja, $\mathrm{RBE}=$ Refinería de Baja Eficiencia, $\mathrm{RAE}=$ Refinería de Alta Eficiencia

no, en porcentaje, utilizando el peso molecular del crudo (CC). Para esto, se obtiene un factor (FR) que relaciona el contenido de carbono y el peso molecular del crudo con el peso molecular del $\mathrm{CO}_{2}$ y el C (44/12). Por otro lado, se utiliza una fórmula para encontrar los barriles de crudo con base en su densidad en grados
API (B). Con el factor de relación FR y la fórmula mencionada anteriormente se obtiene el factor de emisión del $\mathrm{CO}_{2}\left(\mathrm{FECO}_{2}\right)$.

Como auxiliares en estos cálculos se utilizaron los datos de la tabla 5 para obtener el peso molecular de cada crudo (PM) mediante el punto de ebullición promedio molar (PEPM), al mismo tiempo se utilizaron para esta operación el factor de caracterización Watson y la gravedad específica $(k, S)$, el porcentaje en peso de carbono $(\mathrm{wt} \%)$ para calcular el contenido de carbono (CC) y los grados API para calcular los barriles contenidos en una tonelada de crudo (B).

Algoritmo de cálculo para determinar el factor de emisión de $\mathrm{CO}_{2}$ por contenido de carbono:

1) Determinar el peso molecular utilizando las ecuaciones 2 y 3 (Ganji et al., 2010).

$$
P M=\left(4.5673 * 10^{-5}\right)\left(P E M P^{2.1962}\right)\left(S^{-1.0164}\right)
$$

Tabla 4. Consumo de energía procesando crudo Maya con déficit de gasolinas

\begin{tabular}{|c|c|c|c|c|c|c|c|c|c|c|c|}
\hline \multirow{3}{*}{ Refinería } & \multirow{3}{*}{ Escenarios } & \multicolumn{10}{|c|}{ Miles de toneladas de petróleo equivalente (Mtepd) } \\
\hline & & \multicolumn{5}{|c|}{ RBE } & \multicolumn{5}{|c|}{ RAE } \\
\hline & & 2015 & 2020 & 2025 & 2030 & Total & 2015 & 2020 & 2025 & 2030 & Total \\
\hline \multirow{4}{*}{ R1 } & BAU & 24 & 33 & 43 & 55 & 173 & 14 & 19 & 25 & 31 & 99 \\
\hline & RAG-C & 52 & 71 & 91 & 108 & 357 & 30 & 41 & 52 & 62 & 204 \\
\hline & RAG-B & 86 & 115 & 139 & 148 & 543 & 49 & 66 & 79 & 85 & 310 \\
\hline & RAG-A & 108 & 139 & 156 & 157 & 632 & 62 & 80 & 89 & 90 & 361 \\
\hline \multirow{4}{*}{ R2 } & BAU & 21 & 28 & 37 & 47 & 148 & 12 & 16 & 22 & 27 & 86 \\
\hline & RAG-C & 44 & 61 & 78 & 92 & 306 & 26 & 35 & 46 & 54 & 178 \\
\hline & RAG-B & 73 & 99 & 118 & 127 & 464 & 43 & 58 & 69 & 74 & 271 \\
\hline & RAG-A & 93 & 119 & 134 & 134 & 540 & 54 & 70 & 78 & 78 & 315 \\
\hline \multirow{4}{*}{ R3 } & BAU & 18 & 24 & 31 & 39 & 124 & 11 & 15 & 19 & 24 & 76 \\
\hline & RAG-C & 37 & 51 & 66 & 78 & 257 & 23 & 31 & 40 & 48 & 158 \\
\hline & RAG-B & 62 & 83 & 100 & 107 & 391 & 38 & 51 & 61 & 65 & 240 \\
\hline & RAG-A & 78 & 100 & 113 & 113 & 455 & 48 & 62 & 69 & 69 & 279 \\
\hline \multirow{4}{*}{ R4 } & BAU & 14 & 18 & 24 & 31 & 97 & 9 & 12 & 15 & 19 & 61 \\
\hline & RAG-C & 29 & 40 & 31 & 61 & 181 & 18 & 25 & 20 & 38 & 113 \\
\hline & RAG-B & 48 & 65 & 78 & 83 & 304 & 30 & 40 & 49 & 52 & 190 \\
\hline & RAG-A & 61 & 78 & 88 & 88 & 354 & 38 & 49 & 55 & 55 & 221 \\
\hline
\end{tabular}

Nota: $\mathrm{R} 1$ = refinería simple, $\mathrm{R} 2=$ refinería semi compleja, $\mathrm{R} 3=$ refinería compleja y $\mathrm{R} 4=$ refinería muy compleja, $\mathrm{BAU}=$ Escenario Tendencial, RAG-C=Escenario Inflacionario de Referencia, RAG-B=Escenario Inflacionario Bajo, RAG-A=Escenario Inflacionario Alto, RBE=Refinería de Baja Eficiencia, $\mathrm{RAE}=$ Refinería de Alta Eficiencia 
Donde:

$$
\begin{aligned}
& P M \quad=\text { Peso Molecular } \\
& P E P M=\text { Punto de Ebullición Promedio Molar } \\
& S \quad=\text { Gravedad Específica }
\end{aligned}
$$

El PEPM se obtiene al aplicar la siguiente ecuación, k y S se sacan de la tabla 5 .

$$
K=\frac{\sqrt[3]{P E P M}}{S} \text { Obteniendo } \quad P E P M=\left(k^{*} S\right)^{3}
$$

Calcular el contenido de carbono mediante la ecuación 4 (wt\% se consigue de la tabla 5).

$$
C C=\frac{P M^{*} w t \%}{100}
$$

Establecer el factor de relación (FR) empleando el CC y el PM del crudo analizado con el peso molecular de la relación $\mathrm{CO}_{2} / \mathrm{C}(44 / 12)$ utilizando la ecuación 5.

$$
F R=\left(\frac{C C}{P M}\right) * \frac{44}{12}
$$

Usando la gravedad API de la tabla 5 calculamos los barriles de crudo analizado por tonelada ( $\mathrm{t}$ ) utilizando la ecuación 6.

$$
B=\frac{1}{\left(\frac{141.5}{A P I+131.5}\right) * 0.15}
$$

Finalmente se calcula el Factor de Emisión del $\mathrm{CO}_{2}$ utilizando la ecuación 7.

$$
F E C O 2=\frac{F R}{B}
$$

En la tabla 6 se muestran los factores de emisión de $\mathrm{CO}_{2}$, en unidades de masa por unidad de volumen y en unidades de masa por unidad de energía, utilizando los dos procedimientos descritos anteriormente (PICC y cálculo estequiométrico por contenido de carbono).

En la tabla 7 se presentan las emisiones de $\mathrm{CO}_{2}$, procesando crudo Maya considerando solo el déficit de gasolinas en refinerías de baja eficiencia.

\section{Análisis y discusión de resultados}

Se puede observar que dependiendo de las opciones tecnológicas de refinación de petróleo (número de procesos de cada refinería) se tiene un nivel de complejidad diferente en cada caso. De esta forma, a mayor número de procesos (tabla 1), mayor será el grado de complejidad de la refinería, que en este caso es del tipo R4 (full conversion). Si bien cada tipo de proceso tiene su nivel de complejidad, la sumatoria de cada uno de ellos contribuye en el nivel de complejidad total de la refinería. El grado de complejidad total será inversamente proporcional a la capacidad de destilación atmosférica que tenga cada refinería (ecuación 1), y que en este caso corresponde a la de tipo R1, como se puede observar en la tabla 2 y en el ejemplo desarrollado después de dicha ecuación. Los cálculos para los años posteriores a 2015 y para los diferentes tipos de refinerías (R1, R2, R3 y $\mathrm{R} 4)$, se realizaron de la misma manera que en el ejemplo presentado, y siempre se obtienen los mismos resultados en el nivel de complejidad para cada caso.

En la figura 2, se puede decir que las ecuaciones que gobiernan cada recta presentada indican una mayor pendiente en las refinerías de baja eficiencia (RBE). Esto quiere decir que existe un mayor consumo de energía

\begin{tabular}{|c|c|c|c|c|c|c|c|}
\hline & Olmeca & Istmo & Maya & M1 & M2 & M3 & M4 \\
\hline $\begin{array}{l}\text { Factor de } \\
\text { caracterización } \\
\text { Watson (k) }\end{array}$ & ${ }^{*} 12.00$ & ${ }^{*} 11.95$ & ${ }^{*} 11.71$ & 11.88 & 11.87 & 11.83 & 11.82 \\
\hline $\begin{array}{l}\text { Gravedad específica } \\
\text { (S) }\end{array}$ & ${ }^{*} 0.8315$ & ${ }^{*} 0.8584$ & ${ }^{*} 0.9260$ & 0.879 & 0.882 & 0.892 & 0.896 \\
\hline Gravedad API & *38.67 & *33.34 & *21.31 & 29.73 & 29.13 & 27.33 & 26.6 \\
\hline $\begin{array}{l}\text { Porcentaje en peso } \\
\text { de carbono (wt } \%)\end{array}$ & $* * 85.91$ & $* * 85.40$ & $* * 83.96$ & 85.0 & 84.9 & 84.7 & 84.6 \\
\hline
\end{tabular}
por cada unidad de masa alimentada a este tipo de refinerías, en comparación con las de alta eficiencia (RAE). Además, el grado de correlación $\left(\mathrm{R}^{2}\right)$ es mayor en el último tipo de refinerías, es decir, que los datos obtenidos están más apegados a la realidad sobre la información recabada de dichas refinerías y que, efectivamente, las

Tabla 5. Propiedades típicas del petróleo crudo puro y sus mezclas

Fuente: (Ancheyta, 2011); (Ancheyta et al., 2002). M1, M2, M3 y M4 se obtuvieron con base en datos de Ancheyta (2011 y 2002), multiplicando las diferentes relaciones de los crudos para obtener cada una de las mezclas 
Tabla 6. Factores de emisión por tipo de crudo empleando la metodología del PICC y por cálculo estequiométrico por contenido de carbono

\begin{tabular}{lcccc}
\hline & \multicolumn{2}{c}{$\mathrm{t} \mathrm{CO}_{2} / \mathrm{bl}$} & \multicolumn{2}{c}{$\mathrm{t} \mathrm{CO}_{2} /$ tep } \\
\hline Metodología & $\begin{array}{c}\text { Metodología } \\
\text { Contenido de } \\
\text { Carbono }\end{array}$ & $\begin{array}{c}\text { Metodología } \\
\text { PICC }\end{array}$ & $\begin{array}{c}\text { Metodología } \\
\text { Contenido de } \\
\text { Carbono }\end{array}$ \\
\hline OLMECA & 0.405 & 0.414 & 2.96 & 3.03 \\
ISTMO & 0.418 & 0.426 & 3.01 & 3.12 \\
MAYA & 0.446 & 0.451 & 3.09 & 3.30 \\
M1 & 0.426 & 0.433 & 3.03 & 3.17 \\
M2 & 0.427 & 0.435 & 3.03 & 3.18 \\
M3 & 0.432 & 0.439 & 3.05 & 3.21 \\
M4 & 0.433 & 0.44 & 3.05 & 3.22 \\
\hline
\end{tabular}

Nota: (t $\mathrm{CO}_{2} / \mathrm{bl}$ ): Toneladas de bióxido de carbono por barril procesado. ( $\mathrm{CO}_{2} /$ tep): Toneladas de bióxido de carbono por tonelada equivalente de petróleo procesado. M1 (70\% Istmo y $30 \%$ Maya). M2 (65\% Istmo y 35\% Maya). M3 (50\% Istmo y 50\% Maya). M4 (44\% Istmo y 56\% Maya), PICC: Panel Intergubernamental para el Cambio Climático

Tabla 7. Emisiones de bióxido de carbono procesando crudo Maya y considerando refinerías de baja eficiencia

\begin{tabular}{|c|c|c|c|c|c|c|c|c|c|c|c|}
\hline \multirow{3}{*}{ Refinería } & \multirow{3}{*}{ Escenarios } & \multicolumn{10}{|c|}{ Miles de toneladas diarias (Mtd) } \\
\hline & & \multicolumn{5}{|c|}{ Metodología PICC } & \multicolumn{5}{|c|}{ Metodología Contenido de Carbono } \\
\hline & & 2015 & 2020 & 2025 & 2030 & Total & 2015 & 2020 & 2025 & 2030 & Total \\
\hline \multirow{4}{*}{ R1 } & BAU & 75.7 & 101.8 & 133.9 & 168.6 & 534.4 & 76.5 & 103 & 135.4 & 170.5 & 540.4 \\
\hline & RAG-C & 159.9 & 219.3 & 282.4 & 334.3 & 1104.5 & 161.7 & 221.8 & 285.5 & 338 & 1116.8 \\
\hline & RAG-B & 264.5 & 356.7 & 428.2 & 457.7 & 1678.7 & 267.5 & 360.7 & 433 & 462.8 & 1697.5 \\
\hline & RAG-A & 334.9 & 431.2 & 483.3 & 485.3 & 1952.5 & 338.6 & 436 & 488.7 & 490.7 & 1974.4 \\
\hline \multirow{4}{*}{$\mathbf{R} 2$} & BAU & 64.7 & 87.1 & 114.6 & 144.2 & 457.1 & 65.5 & 88.1 & 115.8 & 145.8 & 462.2 \\
\hline & RAG-C & 136.8 & 187.6 & 241.5 & 285.9 & 944.6 & 138.3 & 189.7 & 244.2 & 289.1 & 955.2 \\
\hline & RAG-B & 226.2 & 305 & 366.2 & 391.5 & 1435.7 & 228.8 & 308.5 & 370.3 & 395.9 & 1451.8 \\
\hline & RAG-A & 286.4 & 368.8 & 413.3 & 415 & 1669.9 & 289.6 & 372.9 & 418 & 419.7 & 1688.7 \\
\hline \multirow{4}{*}{ R3 } & BAU & 54.5 & 73.3 & 96.4 & 121.4 & 384.7 & 55.1 & 74.1 & 97.5 & 122.7 & 389 \\
\hline & RAG-C & 115.1 & 157.9 & 203.3 & 240.6 & 795 & 116.4 & 159.7 & 205.5 & 243.3 & 803.9 \\
\hline & RAG-B & 190.4 & 256.7 & 308.2 & 329.5 & 1208.4 & 192.5 & 259.6 & 311.7 & 333.2 & 1221.9 \\
\hline & RAG-A & 241 & 310.4 & 347.9 & 349.3 & 1405.5 & 243.8 & 313.8 & 351.8 & 353.2 & 1421.3 \\
\hline \multirow{4}{*}{ R4 } & BAU & 42.4 & 57.1 & 75.1 & 94.5 & 299.5 & 42.9 & 57.7 & 75.9 & 95.6 & 302.9 \\
\hline & RAG-C & 89.6 & 122.9 & 97.3 & 187.3 & 558.1 & 90.6 & 124.3 & 98.4 & 189.4 & 564.3 \\
\hline & RAG-B & 148.2 & 199.9 & 240 & 256.5 & 940.8 & 149.9 & 202.1 & 242.7 & 259.4 & 951.3 \\
\hline & RAG-A & 187.7 & 241.6 & 270.8 & 272 & 1094.3 & 189.8 & 244.3 & 273.9 & 275 & 1106.5 \\
\hline
\end{tabular}

Nota: $\mathrm{R} 1$ = refinería simple, R2 = refinería semi compleja, R3= refinería compleja y $\mathrm{R} 4=$ refinería muy compleja, $\mathrm{BAU}=$ Escenario Tendencial, IR=Escenario Inflacionario de Referencia, IB=Escenario Inflacionario Bajo, IA=Escenario Inflacionario Alto, PICC=Panel Intergubernamental para el Cambio Climático 
de alta eficiencia consumen menos energía por cada unidad de masa alimentada a las mismas.

De los cálculos realizados utilizando los datos de las tablas 3 y datos para un escenario BAU como ejemplo (Granados et al., 2013), se puede inferir que la cantidad de energía consumida necesaria para que una refinería cubra una demanda de petrolíferos (gasolinas) es mínima, siempre y cuando se utilice una de tipo muy compleja (full conversion), ya que la tendencia de consumo de energía (figura 3), es a la baja conforme aumenta el grado de complejidad, esto, para un año en particular.

De la misma forma y utilizando el mismo procedimiento anterior en la tabla 4 , se muestran los resultados de todos los cálculos para diferentes años procesando solamente crudo Maya en las opciones de alta y baja eficiencia. Analizando esta tabla, se puede ver que independientemente del año, o de si es con alta o baja eficiencia, la tendencia general es que a mayor grado de complejidad menor será el consumo de energía para todos los escenarios.

Con respecto a los factores de emisión de bióxido de carbono, la tabla 6 muestra una diferencia en resultados de menos de $2 \%$ con respecto a los valores obtenidos con la metodología del PICC. Esto quiere decir que los cálculos realizados y mostrados en el cuerpo de este trabajo son consistentes.

Para finalizar, se observa en la tabla 7, que independientemente del año de la metodología y de si se realiza con alta o baja eficiencia, las emisiones de bióxido de carbono tienen una tendencia a la baja conforme aumenta el grado de complejidad de la refinería para todos los escenarios.

\section{Conclusiones}

En el presente trabajo el objetivo principal fue conocer el impacto de consumo de energía por unidad de crudo procesado y las emisiones de $\mathrm{CO}_{2}$, utilizando la metodología del PICC, así como el cálculo estequiométrico con base en el contenido de carbono, el cual se cumplió de manera formal. De acuerdo con los resultados, el consumo de petrolíferos para los próximos 20 años tenderá a la alta independientemente del desarrollo de tecnologías en energías alternas que se implementen en ese periodo. En especial el consumo de gasolinas implicará un aumento en los niveles de refinación de crudos, lo que a su vez, tendrá un impacto en el tipo de refinería a utilizar que cumpla con todos los requisitos para su viabilidad en términos de costos y demanda del mercado interno. Esto mismo tendrá como consecuencia inherente un aumento en el tipo de emisiones de gases de efecto invernadero producto del mismo proceso de refinación (BP, 2013).
En este estudio se encontró que el nivel de complejidad por tipo de refinería a utilizar juega un papel importante en la relación costo-beneficio en cuanto al costo de la refinería y el nivel de emisiones que genera la misma. El resultado de este trabajo indica que para una demanda en específico de gasolinas, el elegir una refinería de mayor complejidad es costeable en cuanto a que el consumo de energía es mucho menor para su funcionamiento, en comparación con las menos complejas. Pero, esta misma complejidad implicará un mayor consumo de energía (aunque ligero) para la operación de dicha refinería. Al hacer el balance energético para cumplir una demanda de gasolinas, se puede ver que conviene a largo plazo la utilización de una refinería de mayor complejidad ( $\mathrm{R} 4)$.

Utilizar refinerías muy complejas será entonces el "costo" que se tiene que pagar por emitir menos contaminantes a la atmósfera, lo cual a largo plazo trae un balance positivo en el costo-beneficio, porque se cubre la demanda de petrolíferos de alta demanda y se cumple con la petición de bonos de carbono impuestos por los organismos internacionales (United Nations, 2014). Los factores de emisión calculados con la metodología de cálculo estequiométrico a partir del Contenido de Carbono, son similares a los encontrados por la metodología del PICC e incluso, materialmente iguales a los reportados por la EPA (2014). Esto quiere decir que los resultados mostrados en este trabajo son de alguna manera consistentes para poder utilizarse posteriormente en algún otro tipo de investigación de esta área.

\section{Referencias}

Ancheyta J. Modeling and simulation of catalytic reactors for petroleum refining, Nueva Jersey, USA, John Wiley \& Sons, Inc., 2011[en línea][fecha de consulta: 1 de septiembre de 2014].

Disponible en: http://www.google.com.mx/url?sa=t\&rct $=\mathrm{j} \& \mathrm{q}=\& \mathrm{esrc}=\mathrm{s} \&$ source $=$ web \& $\mathrm{cd}=2 \& \mathrm{ved}=0 \mathrm{CDQQFj} A B \& \mathrm{u}$ rl=http $\% 3 \mathrm{~A} \% 2 \mathrm{~F} \% 2 \mathrm{Fbib}$. convdocs.org $\% 2 \mathrm{Fv} 24505 \% 2 \mathrm{~F} \% 3$ Fdownload\%3D1\&ei=fr3WUr6fAZOEoQTOzIDYCg\&us $\mathrm{g}=\mathrm{AFQjCNH5QN4xYXPIaiD9YaXIp1RbGDYE6g}$.

Ancheyta J., Centeno G., Trejo F., Marroquín G., García J.A.,Tenorio E., Torres A. Extraction and characterization of asphaltenes from different crude oils and solvents. Energy \& Fuels, volumen 16, 2002: 1121-1127.

Baird C.T. Handbook $\&$ database of petroleum refinning yields, Arizona, USA, HPI Consultants, INC, Dept. of Commerce Schedule B Commodity Number: 4901.99.0050, 1996, pp. 61, 79, 86.

Bauer M., Mar E., Elizalde A. Transport and energy demand in México: the personal income shock. Energy Policy, volumen 31 (número 14), noviembre, 2003: 1475-1480. 
BP statistical review of world energy, junio 2013, Orb solutions, Londres, 2013 [en línea] [fecha de consulta: 29 de agosto de 2014]. Disponible en: http://www.bp.com/content/dam/bp/ pdf/statistical-review/statistical_review_of_world_energy_2013.pdf

BP statistical review of world energy junio 2013. Carbon dioxide emissions, orb solutions, Londres, 2013 [en línea] [fecha de consulta: 29 de agosto de 2014]. Disponible en: http://www. bp.com/content/dam/bp/pdf/statistical-review/statistical_review_of_world_energy_2013.pdf.

Enerdata, Global energy consumption statistical yearbook 2013, EMEA, Londres, 2013 [en línea] [fecha de consulta: 29 de agosto de 2014]. Disponible en: http://yearbook.enerdata.net/ energy-consumption-data.html.

Environment Protection Agency Industry (EPA). Barrels of oil consumed, USA, 2014 [en línea] [fecha de consulta: 2 de septiembre de 2014]. Disponible en: http://www.epa.gov/cleanenergy/energy-resources/refs.html.

Environment Protection Agency. Industry (EPA) 1997. Profile for the petroleum refinery neshap [en línea]. Disponible en: http://www.epa.gov/ttnecas1/regdata/IPs/Petroleum\%20Refinery \%20(Sulfur\%20Recovery\%20Units, \%20Catalytic\%20 Crackin.pdf.

Granados H.E., López A.X., Bravo A.H., Sosa E.R. Refinación de petróleo y su impacto económico-tecnológico para la producción de gasolinas en México al 2030. Revista Ingeniería Investigación y Tecnología, volumen XIV (número 4), octubre-noviembre, 2013: 475-487.

Grid-Arendal. Consumo de energía, desarrollo económico y emisiones de $\mathrm{CO}_{2}$ en algunos países de América Latina, Norway, 2014 [en línea] [fecha de consulta: 2 de septiembre de 2014]. Disponible en: http://www.grida.no/publications/vg/lacsp/ page/2784.aspx

Ganji H., Zahedi S., Ahmadi-Marvast M., Kananpanah S., Sadi M., Shokri S. Determination of suitable feedstock for refineries utilizing LP and NLP models. International Journal of Chemical Engineering and Applications, volumen 1 (número 3), octubre de 2010: 225-229.

International Energy Agency (IEA). $\mathrm{CO}_{2}$ emissions from fuel combustion, Paris, 2010a [en línea] [fecha de consulta: 2 de septiembre de 2014]. Disponible en: http://www.oecd-ilibrary. org/docserver/download/6110121e.pdf?expires=1377043092\& id=id\&accname $=$ oid006814\&checksum $=$ A975759A4CD64D AD0535800561610872.

International Energy Agency (IEA). Energy statistics of nonOECD countries, Paris, 2011a [en línea] [fecha de consulta: 2 de septiembre de 2014]. Disponible en: http://www.oecd-ilibrary.org/docserver/download/6111151e.pdf?expires=137704 0402\&id=id\&accname $=$ oid006814\&checksum $=$ F4179F6A047E B7A9EEC9D24DD685419D.

International Energy Agency (IEA). Energy statistics of OECD countries, Paris, 2011b [en línea] [fecha de consulta: 2 de septiembre de 2014]. Disponible en: http://www.cne.es/cgibin/BRSCGI.exe?CMD=VEROBJ\&MLKOB=577558661616.

International Energy Agency (IEA). $\mathrm{CO}_{2}$ emissions from fuel combustion, Paris, 2011c [en línea] [fecha de consulta: 2 de septiembre de 2014]. Disponible en: http://www.oecd-ilibrary. org/docserver/download/6111121e.pdf?expires $=1393545823 \&$ id $=i d \&$ accname $=$ oid006814\&checksum $=64532472$ F163ED20A FC8BC64C9097B50

International Energy Agency (IEA). Energy statistics of nonOECD countries, Paris, 2012a [en línea] [fecha de consulta: 2 de septiembre de 2014]. Disponible en: http://www.oecd-ilibrary.org/docserver/download/6112121e.pdf?expires=137704 0550\&id=id\&accname $=$ oid006814\&checksum $=$ F01C1EA08852 0720FC439699FEB08B51

International Energy Agency (IEA). Energy statistics of OECD countries, Paris, 2012b [en línea] [fecha de consulta: 2 de septiembre de 2014]. Disponible en: http://www.oecd-ilibrary. org/docserver/download/6112091e.pdf?expires=1377041598\& id=id\&accname $=$ oid006814\&checksum $=$ A73EBD32BAF56514 935A5EE0F9EA96E6.

International Energy Agency (IEA). $\mathrm{CO}_{2}$ emissions from fuel combustion, Paris, 2012c [en línea] [fecha de consulta: 2 de septiembre de 2014]. Disponible en: http://www.oecd-ilibrary. org/docserver/download/6111121e.pdf?expires $=1377043346 \&$ id $=$ id\&accname $=$ oid006814\&checksum $=16$ B54314C955C912E F2382F158A85C42.

International Energy Agency (IEA). Energy statistics of nonOECD countries, Paris, 2013a [en línea] [fecha de consulta: 2 de septiembre de 2014]. Disponible en: http://www.oecd-ilibrary.org/docserver/download/6113191e.pdf?expires=137703 9918\&id=id\&accname=oid006814\&checksum=1AEE2D3EC92 C277AD98ECECD3BFE16A7.

International Energy Agency (IEA). Energy statistics of OECD countries, Paris, 2013b [en línea] [fecha de consulta: 2 de septiembre de 2014]. Disponible en: http://www.oecd-ilibrary. org/docserver/download/6113161e.pdf?expires $=1377041889 \&$ id=id\&accname $=$ oid006814\&checksum $=$ CD02CC895F5C4FC1 DEBE7D602783835D.

International Energy Agency (IEA). Working together to ensure reliable, affordable and clean energy, Paris 2013c [en línea] [fecha de consulta: 2 de septiembre de 2014]. Disponible en: http://www.iea.org/newsroomandevents/news/2012/may/ name,27216,en.html.

Intergovernmental Panel on Climate Change (IPCC) 2014. Directrices del IPCC de 2006 para los inventarios nacionales de gases de efecto invernadero, Switzerland, 2013b [en línea] [fecha de consulta: 17 de septiembre de 2014]. Disponible en: http://www.ipcc-nggip.iges.or.jp/public/2006gl/spanish/ vol2.html.

Ozren O. Oil refineries in the 21st century: energy efficient, cost effective, environmentally benign, Serbia, ediciones WILEY-VCH verlag GmbH \& Co. kGa, Weinheim, 2005, pp. 8-10. 
United Nations. Framework convention on climate change, Germany, 2014 [en línea] [fecha de consulta: 2 de septiembre de 2014]. Disponible en: https://unfccc.int/2860.php.

\begin{abstract}
Este artículo se cita:
Citación estilo Chicago

Granados-Hernández, Elías, Humberto Bravo-Álvarez, Rodolfo Sosa-Echeverría, Xicoténcatl López-Andrade, Claudia García-Landa, Pablo Sánchez-Álvarez. Consumo de energía y emisiones de bióxido de carbono del sector refinación de petróleo en México de 2015 a 2030. Ingeniería Investigación y Tecnología, XVI, 04 (2015): 503-513.

\section{Citación estilo ISO 690}

Granados-Hernández E., Bravo-Álvarez H., Sosa-Echeverría R., López-Andrade X., García-Landa C., Sánchez-Álvarez P. Consumo de energía y emisiones de bióxido de carbono del sector refinación de petróleo en México de 2015 a 2030. Ingeniería Investigación y Tecnología, volumen XVI (número 4), octubre-diciembre 2015: 503-513.
\end{abstract}

\section{Semblanzas de los autores}

Elías Granados-Hernández. Realizó su doctorado en ingeniería en la UNAM con la especialidad en planeación energética y contaminación ambiental en el Centro de Investigación en Energía (CIE). Desde 1996 se desempeña como académico en el Laboratorio de Ingeniería Ambiental del Centro Tecnológico Aragón de la UNAM, realiza investigación en el área de estudios energéticos y ambientales.

Humberto Bravo-Álvarez. Es investigador en el Centro de Ciencias de la Atmósfera (CCA) de la UNAM, donde se desempeña como jefe de la Sección de Contaminación Ambiental desde 1977. Su área de interés por más de 45 años ha sido la contaminación y la ingeniería ambiental. Ha sido profesor de asignatura en la maestría en ingeniería ambiental en la División de Estudios de Posgrado de la Facultad de Ingeniería (DEPFI) de la UNAM desde 1988. Es miembro honorario de la Air and Waste Management Association. Cuenta con la certificación internacional Qualified Environmental Professional (QEP) del Institute of Professional Environmental Practice. Es miembro del Sistema Nacional de Investigadores.

Rodolfo Sosa-Echeverría. Realizó su doctorado en ingeniería en la UNAM. Labora como investigador en la Sección de Contaminación Ambiental del CCA desde 1986. Desde 1988 a la fecha ha impartido la cátedra de muestreo y análisis del aire en el posgrado en ingeniería ambiental de la Facultad de Ingeniería. Fue presidente del capítulo México de la Air and Waste Management Association, de 1991 a 1993. Cuenta con la certificación internacional Qualified Environmental Professional (QEP) del Institute of Professional Environmental Practice. Es miembro del Sistema Nacional de Investigadores.

Xicoténcatl López-Andrade. Realizó su doctorado en ingeniería de procesos en la UNAM, un posdoctorado en Barcelona, España en el laboratorio de nanotecnología aplicado al área de ciencias ambientales. Ha sido profesor de asignatura en la carrera de ingeniería química.

Claudia García-Landa. Realizó su doctorado en psicología ambiental. Su principal línea de investigación es el consumo energético y contaminación ambiental.

Pablo Sánchez-Álvarez. Realizó la licenciatura en biología en la Facultad de Ciencias de la UNAM. Actualmente labora en la Sección de Contaminación Ambiental del CCA. 